03

\title{
Кольцевые волны на поверхности жидкости в кулоновском поле
}

\author{
() С.О. Ширяева, А.И. Григорьев \\ Ярославский государственный университет им. П.Г. Демидова, \\ 150000 Ярославль, Россия \\ e-mail: shir@uniyar.ac.ru
}

Поступило в Редакцию 25 декабря 2017 г.

В окончательной редакции 18 февраля 2019 г.

Принято к публикации 25 февраля 2019 г.

\begin{abstract}
Исследованы закономерности реализации неустойчивости кольцевых волн на поверхности электропроводной жидкости в кулоновском поле. Выведено соотношение для связи частоты кольцевых капиллярногравитационных волн на поверхности проводящей жидкости в неоднородном электростатическом поле точечного заряда с физическими параметрами задачи и с аналогом волнового числа. По аналогии с полевым параметром Тонкса-Френкеля определен полевой параметр, характеризующий устойчивость поверхности проводящей жидкости в неоднородном кулоновском поле. Найдено, что критические условия реализации неустойчивости кольцевых волн на поверхности электропроводной жидкости в кулоновском поле менее жестки, чем критические условия реализации неустойчивости Тонкса-Френкеля.
\end{abstract}

Ключевые слова: кольцевые волны, кулоновское поле.

DOI: $10.21883 /$ JTF.2019.08.47890.2612

\section{Введение}

В ряде экспериментальных работ (см., например, $[1,2])$ исследовался электрический разряд между поверхностью жидкости, находящейся в цилиндрическом сосуде, и противоэлектродом (стержнем), расположенным на конечном расстоянии над жидкостью на оси симметрии. Картина разряда в такой установке весьма интересна и нуждается в теоретическом осмыслении. Рельеф свободной поверхности жидкости напоминает рельеф поверхности жидкости при реализации неустойчивости Тонкса-Фрекеля $[3,4]$ (см., например, фотографии в [5]), но в отличие от последней несет элементы осевой симметрии. Волны, возникающие на поверхности жидкости, являются кольцевыми.

С кольцевыми волнами в реальных условиях приходится встречаться регулярно в макромасштабах: при падении тел различной природы на водную поверхность рек, озер, морей и океанов, и в микромасштабах: от падения на водную поверхность капель дождя или от падения на поверхность чая в чашке или в блюдце капли чая из чайника (см., например, [6--11] и литературу там). Кольцевые волны появляются и в бассейнах цилиндрической формы, и в жидком плоском мениске на торце труб кольцевого сечения, заполненных жидкостью, из-за наличия граничных условий на твердых стенках $[12,13]$, в этой связи данный объект неоднократно исследовался как теоретически (см., например, [13,14], так и экспериментально [15].

В итоге складывается впечатление, что кольцевые волны только формой отличаются от плоских, которые исследованы значительно подробней (см., например, монографии $[16,17]$ и указанную в них литературу). Но как отмечено в [14], амплитуда кольцевых волн не постоянна, а уменьшается с увеличением расстояния от места возникновения. Также у них непостоянна длина волны, т.е. понятие „волнового числа“ для них не определено. Сказанное означает, что к данной ситуации и понятие дисперсионного уравнения (уравнения, связывающего между собой частоты и волновые числа волн) применимо весьма условно.

Различие в свойствах плоских и кольцевых волн в идеальной жидкости связано с тем, что плоские волны в декартовой системе координат одномерны, и для них сумма кинетической и потенциальной энергии сохраняется при сохранении их формы. Кольцевые же волны в той же системе координат двумерны, их амплитуда снижается, а скорость распространения уменьшается по мере увеличения расстояния от места возникновения в силу закона сохранения энергии.

Нижеследующее исследование проведем на примере жидкости в круглом бассейне, над которой помещен точечный заряд. Берется точечный заряд, а не стержень, как в экспериментах $[1,2]$, чтобы максимально упростить вид электрического поля, сохранив его осевую симметрию и неоднородность, так как электростатическое поле стержня, поддерживаемого при некотором потенциале, имеет весьма сложный вид [18] (задача 74), что неоправданно загромоздит расчеты.

\section{Постановка задачи}

Пусть дана идеальная, несжимаемая, идеально проводящая бесконечно глубокая жидкость с массовой плотностью $\rho$ и коэффициентом поверхностного натяжения $\sigma$, ограниченная твердой цилиндрической стенкой, радиуса $R$. Введем цилиндрическую систему координат $(r, \varphi, z)$, начало которой совмещено с центром 
свободной поверхности бассейна. Ось $O z$ направлена вертикально вверх, а плоскость $z=0$ совпадает с плоской (в отсутствии электрического поля) равновесной свободной поверхностью жидкости. Вся система находится в поле силы тяжести g. На высоте $L$ (примем для определенности, что $L<R$ ) от поверхности жидкости, над центром бассейна (на оси $O z$ ), закреплен точечный заряд $q$. Будем искать равновесную форму свободной поверхности жидкости и исследовать ее на устойчивость. Рассмотрение ограничим осесимметричными волнами.

Следует отметить, что в жидкости из-за теплового движения молекул [4] генерируется капиллярное волновое движение весьма малой (тепловой) амплитуды с характерной высотой гребней $|\xi|: \sqrt{\kappa T / \sigma}$, где $\kappa-$ постоянная Больцмана; $T$ - абсолютная температура. При температурах порядка комнатной для простых и органических жидкостей, воды и для жидких металлов амплитуда волн меньше ангстрема. Это волновое движение практически бесконечно малой амплитуды формирует искажение равновесной поверхности жидкости вида $\xi(\theta, t)$. Причем $\max |\xi(r, t)| \ll a \equiv \sqrt{\sigma / \rho g}=L$, где $a$ - капиллярная постоянная жидкости [11]. Отношение $|\xi(r, t)| / a$ примем в качестве первого малого параметра задачи $\varepsilon$.

Уравнение свободной поверхности жидкости представим в виде

$$
F(r, z, t) \equiv z-h(r)-\xi(r, t)=0
$$

где $h(r)$ - функция, описывающая равновесную поверхность.

Математическая формулировка задачи имеет вид

$$
\begin{gathered}
\frac{\partial \mathbf{V}(r, z, t)}{\partial t}+(\mathbf{V}(r, z, t) g \nabla) \mathbf{V}(r, z, t) \\
=-\frac{1}{\rho} \nabla P(r, z, t)+\mathbf{g} ; \operatorname{div} \mathbf{V}(r, z, t)=0 ; \\
\operatorname{rot} \mathbf{E}(r, z, t)=0 ; \operatorname{div} \mathbf{E}(r, z, t)=0 ; \\
r \rightarrow-\infty: \mathbf{V}(r, z, t) \rightarrow 0 ; z \rightarrow+\infty: \mathbf{E}(r, z, t) \rightarrow 0 ; \\
r \rightarrow 0: \mathbf{V}(r, z, t)<\infty ; \mathbf{E}(r, z, t)<\infty ; \\
r=R: \mathbf{n}(\mathbf{r}, t) g \mathbf{V}(r, z, t)=0 ; \\
z=h(r)+\xi(r, t):\left(\frac{\partial F(\mathbf{r}, t)}{\partial t}+\mathbf{V}(r, z, t) g \nabla F(\mathbf{r}, t)\right)=0 ; \\
P(r, z, t)-P_{a t m}+P_{E}(r, t)=P_{\sigma}(r, t) ; E_{\tau} \equiv \boldsymbol{\tau} g \mathbf{E}=0 .
\end{gathered}
$$

где $\mathbf{V}(r, z, t)$ и $P(r, z, t)$ - поля скоростей и гидродинамического давления в жидкости; $\mathbf{n}(r, t)$ и $\boldsymbol{\tau}(r, t)-$ орты нормали и касательной к возмущенной поверхности жидкости; $P_{\sigma}(r, t)$ - давление сил поверхностного натяжения, $P_{E}(r, t)$ - давление электрического поля; $P_{a t m}$ - внешнее постоянное давление.

\section{Скаляризация и линеаризация задачи}

Будем решать задачу (1) в идеализации потенциального течения, в рамках которой скорость представляется как градиент гидродинамического потенциала $\psi(r, z, t)$ [19], имеющего в возможных безразмерных переменных тот же порядок малости, что и безразмерное возмущение поверхности $\xi(r, t)$, т.е. параметр $\varepsilon$ (весь анализ в настоящей работе будет вестись в размерных переменных).

Выпишем решаемую задачу в линейном по $\varepsilon$ приближении. Напряженность электрического поля представим через электростатический потенциал $\Phi(r, z, t)[20]$. В итоге будем иметь

$$
\mathbf{V}(r, z, t) \equiv \nabla \psi(r, z, t) ; \mathbf{E}(r, z, t)=-\nabla \Phi(r, z, t),
$$

где $\psi(r, z, t)$ и $\Phi(r, z, t)$ удовлетворяют уравнениям Лапласа:

$$
\Delta \psi(r, z, t)=0 ; \Delta \Phi(r, z, t)=0
$$

Интегрируя уравнение Эйлера, сразу получим выражение для гидродинамического давления в жидкости

$$
P(r, z, t)=P_{0}-\rho\left(\frac{\partial \psi(r, z, t)}{\partial t}+\frac{1}{2}(\nabla \partial \psi(r, z, t))^{2}\right)-g \rho z .
$$

Граничные условия в терминах потенциалов примут вид

$$
\begin{gathered}
z \rightarrow-\infty: \psi(r, z, t) \rightarrow 0 ; z \rightarrow+\infty: \Phi(r, z, t) \rightarrow 0 ; \\
r \rightarrow 0:|\psi(r, z, t)|<\infty ;|\Phi(r, z, t)|<\infty ; \\
r=R: \frac{\partial \psi(r, z, t)}{\partial r}=0 ; \\
z=h(r)+\xi(r, t): \\
-\frac{\partial \xi(r, t)}{\partial t}+(\nabla \psi(r, z, t), \nabla F(r, t))=0 ; \Phi=\mathrm{const} ; \\
P_{0}-\rho \frac{\partial \psi(r, z, t)}{\partial t}-g \rho(h(r, t)+\xi(r, t))-P_{a t m} \\
+P_{E}(r, t)=P_{\sigma}(r, t) ; \\
P_{E(r, z, t)}=(\nabla \Phi(r, t))^{2} / 8 \pi ; \\
P_{\sigma}(r, t)=\sigma \cdot \operatorname{div} \mathbf{n}(r, t) ; \mathbf{n}(r, t)=\nabla F(\mathbf{r}, t) /\left.\nabla R(\mathbf{r}, t)\right|_{F(\mathbf{r}, \mathbf{t})=0} .
\end{gathered}
$$

Гидродинамический и электростатический потенциалы разложим по порядкам малости, т.е. по степеням малого параметра $\varepsilon$ :

$\psi(r, z, t)=\psi^{(1)}(r, z, t) ; \Phi(r, z, t)=\Phi^{(0)}(r, z)+\Phi^{(1)}(r, z, t)$.

Здесь верхний индекс в скобках обозначает порядок малости. 


\section{Задача нулевого порядка малости по $\varepsilon$}

Выпишем задачу (2) в нулевом порядке малости

$$
\begin{gathered}
\Delta \Phi^{(0)}(r, z)=0 ; z \rightarrow+\infty: \Phi^{(0)}(r, z) \rightarrow 0 ; \\
z=h(r): \Phi^{(0)}(r, z)=0 ; \\
P_{0}-g \rho h(r)-P_{a t m}+P_{E}^{(0)}(r)=P_{\sigma}^{(0)}(r), \\
P_{E}^{(0)(r)}=\left.\frac{\left(\left(\partial_{z} \Phi^{(0)}(r, z)\right)^{2}+\left(\partial_{r} \Phi^{(0)}(r, z)\right)^{2}\right)}{8 \pi}\right|_{z=h(r)} ; \\
P_{\sigma}^{(0)}(r)=-\frac{\sigma\left[h^{\prime}(r)+\left(h^{\prime}(r)\right)^{3} r \cdot h^{\prime \prime}(r)\right]}{r\left(1+h^{\prime}(r)^{2}\right)^{3 / 2}} .
\end{gathered}
$$

Искажение равновесной плоской свободной поверхности жидкости связано с отрицательным давлением на поверхность существующего над ней неоднородного электростатического поля. Величина этого искажения (в безразмерных переменных) должна иметь тот же порядок малости, что и безразмерное давление электрического поля точечного заряда:

$$
\frac{\max |h(r)|}{R}: P_{E}^{(0)}(r) \cdot \frac{R}{\sigma} .
$$

Будем полагать, что отклонение равновесной формы поверхности жидкости от плоскости мало. Введем второй безразмерный параметр $\eta \equiv \frac{\max |h(r)|}{R}$, и будем искать равновесную форму свободной поверхности в виде разложения по $\eta$.

Поскольку безразмерное давление электрического поля : $\eta$, то, следовательно, безразмерные величины напряженности и потенциала электрического поля будут $\sim \sqrt{\eta}$.

Разложим задачу (3) по степеням параметра $\eta$, полагая, что

$$
h(r): \eta ; \quad \Phi^{(0)}(r, z): \sqrt{\eta} .
$$

Используя (4), разобьем задачу (3) по порядкам $\eta^{0}$, $\eta^{1 / 2}, \eta^{1}$.

Нулевой порядок малости позволяет определить константу интегрирования $P_{0}=P_{a t m}$ записать распределение гидродинамического давления в жидкости в равновесном состоянии $P^{(0)}(r, z)$ :

$$
P^{(0)}(r, z)=-g \rho z+P_{a t m} .
$$

Задача порядка $\eta^{1 / 2}$ позволяет определить потенциал электрического поля точечного заряда, находящегося на расстоянии $L$ над плоской поверхностью проводящей жидкости. Ее решение несложно найти методом электростатического отражения:

$$
\Phi^{(0)}(r, z)=q\left(\frac{1}{\sqrt{(L-z)^{2}+r^{2}}}-\frac{1}{\sqrt{(L+z)^{2}+r^{2}}}\right) ;
$$
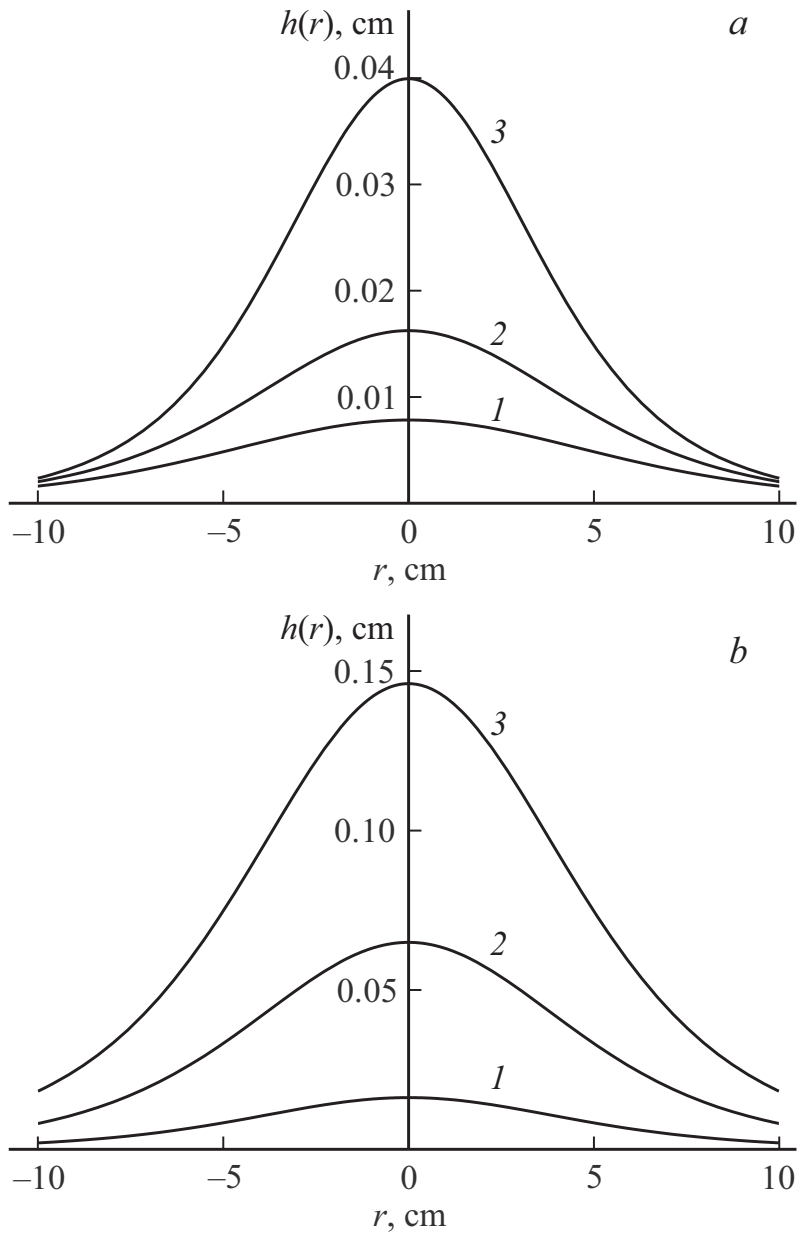

Рис. 1. $a-$ равновесные поверхности, построенные для различных расстояний от заряда до поверхности жидкости. Рассчитано при $\rho=10^{-3} \frac{\mathrm{kg}}{\mathrm{cm}^{3}}, g=0.98 \frac{\mathrm{m}}{\mathrm{s}^{2}}, q=0.33 \mu \mathrm{C}$. $1-L=0.12 \mathrm{~m}, 2-L=0.1 \mathrm{~m}, 3-L=0.08 \mathrm{~m} . b-$ равновесные поверхности, построенные для различных величин зарядов. Рассчитано при $\rho=10^{-3} \frac{\mathrm{kg}}{\mathrm{cm}^{3}}, g=0.98 \frac{\mathrm{m}}{\mathrm{s}^{2}}, L=0.1 \mathrm{~m}$, $1-q=0.33 \mu \mathrm{C}, 2-q=0.66 \mu \mathrm{C}, 3-q=0.99 \mu \mathrm{C}$.

В первом порядке из динамического граничного условия получим дифференциальное уравнение для нахождения функции $h^{\prime}(r)$ :

$$
\sigma h^{\prime \prime}(r)+\sigma \frac{1}{r} h^{\prime}(r)-g \rho h(r)+\frac{L^{2} q^{2}}{2 \pi\left(L^{2}+r^{2}\right)^{3}}=0 .
$$

В пренебрежении капиллярными силами решение (5) легко находится

$$
h(r)=\frac{L^{2} q^{2}}{2 \pi g \rho\left(L^{2}+r^{2}\right)^{3}} .
$$

На рис. 1 изображены графики зависимости $h(r)$, построенные при различных фиксированных значениях $L$ и $q$.

Используя решение (6), оценим для воды $\rho=10^{-3} \frac{\mathrm{kg}}{\mathrm{cm}^{3}}, \quad \sigma=0.0723 \frac{\mathrm{N}}{\mathrm{m}} \quad$ абсолютную величину 
отношений первого и второго слагаемых в выражении (5) к третьему, приняв $L=0.1 \mathrm{~m}$.

Проведенные расчеты показывают, что эти отношения не превышают $1 \%$, следовательно, пренебрежение первыми двумя слагаемыми в выражении (5) обосновано.

\section{Задача первого порядка по $\varepsilon$}

Собирая компоненты первого порядка малости по $\varepsilon$, получим задачу, определяющую временную эволюцию возмущения свободной поверхности жидкости:

$$
\begin{aligned}
& \Delta \psi^{(1)}(r, z, t)=0 ; \Delta \Phi^{(1)}(r, z, t)=0 ; \\
& z \rightarrow-\infty: \psi^{(1)}(r, z, t) \rightarrow 0 ; z \rightarrow+\infty: \Phi^{(1)}(r, z, t) \rightarrow 0 ; \\
& r \rightarrow 0:\left|\psi^{(1)}(r, z, t)\right|<\infty ;\left|\Phi^{(1)}(r, z, t)\right|<\infty \text {; } \\
& r=R: \frac{\partial \psi^{(1)}(r, z, t)}{\partial r}=0 ; \\
& z=h(r): \\
& -\partial_{t} \xi(r, t)+\left(\partial_{z} \psi^{(1)}(r, z, t)-h^{\prime}(r) \partial_{r} \psi^{(1)}(r, z, t)\right)=0 ; \\
& \left(\Phi^{(1)}(r, z, t)+\xi(r, t) \cdot \partial_{z} \Phi^{(0)}(r, z)\right)=0 ; \\
& P^{(1)}(r, t)+P_{E}^{(1)}(r, t)=P_{\sigma}^{(1)}(r, t) ; \\
& P^{(1)}(r, z, t)=-g \rho \cdot \xi(r, t)-\rho \cdot \partial_{t} \psi^{(1)}(r, z, t) ; \\
& P_{E}^{(1)}(r, t)=\frac{1}{4 \pi}\left(\xi(r, t) \cdot \partial_{z} \Phi^{(0)}(r, z) \cdot \partial_{z, z} \Phi^{(0)}(r, z)\right. \\
& +\xi(r, t) \cdot \partial_{r} \Phi^{(0)}(r, z) \cdot \partial_{r, z} \Phi^{(0)}(r, z) \\
& +\partial_{z} \Phi^{(0)}(r, z) \cdot \partial_{z} \Phi^{(1)}(r, z, t) \\
& \left.+\partial_{r} \Phi^{(0)}(r, z) \cdot \partial_{r} \Phi^{(1)}(r, z, t)\right) ; \\
& P_{\sigma}^{(1)}(r, t)=-\frac{\sigma}{\left(1+h^{\prime}(r)^{2}\right)^{3 / 2}}\left[\left(\partial_{r, r} \xi(r, t)\right.\right. \\
& \left.+\left(\frac{1}{r}-\frac{3 h^{\prime}(r) h^{\prime \prime}(r)}{1+h^{\prime}(r)^{2}}\right) \partial_{r} \xi(r, t)\right] .
\end{aligned}
$$

\section{Линеаризация по $\eta$ задачи первого порядка малости по $\varepsilon$}

Очевидно, что уравнения Лапласа для $\psi_{1}(r, z, t)$ и $\Phi(r, z, t)$, граничные условия в нуле и на бесконечности, а также на твердой стенке не изменятся. Граничные условия на свободной поверхности жидкости после процедуры линеаризации по $\eta$ примут следующий вид:

кинематическое:

$$
\begin{aligned}
& z=0:-\partial_{t} \xi(r, t)+\left(\partial_{z} \psi^{(1)}(r, z, t)\right. \\
& \left.+h(r) \partial_{z, z} \psi^{(1)}(r, z, t)-h^{\prime}(r) \partial_{r} \psi^{(1)}(r, z, t)\right)=0
\end{aligned}
$$

динамическое:

$$
\begin{aligned}
& -g \rho \xi(r, t)-\rho\left(\partial_{t} \psi^{(1)}(r, z, t)+h(r) \partial_{z, t} \psi^{(1)}(r, z, t)\right) \\
& +\frac{L q \partial_{z} \Phi^{(1)}(r, z, t)}{2 \pi\left(L^{2}+r^{2}\right)^{3 / 2}}+\sigma\left(\partial_{r, r} \xi(r, t)+\frac{1}{r} \partial_{r} \xi(r, t)\right)=0
\end{aligned}
$$

условие эквипотенциальности:

$$
\Phi^{(1)}(r, z, t)+\xi(r, t) \partial_{z} \Phi^{(0)}(r, z)=0 .
$$

\section{Исследование на устойчивость}

Решения уравнений Лапласа для $\psi^{(1)}(r, z, t)$ и $\Phi^{(1)}(r, z, t)$, удовлетворяющие граничным условиям на стенках бассейна, в цилиндрических координатах с учетом осевой симметрии, будут иметь вид

$$
\begin{gathered}
\psi^{(1)}(r, z, t)=\sum_{n=1}^{\infty} B_{n}(t) \exp \left(\frac{\mu_{n}}{R} z\right) J_{0}\left(\frac{\mu_{n}}{R} r\right) ; \\
\Phi^{(1)}(r, z, t)=\sum_{n=1}^{\infty} A_{n}(t) \exp \left(-\frac{\mu_{n}}{R} z\right) J_{0}\left(\frac{\mu_{n}}{R} r\right) ;
\end{gathered}
$$

где $J_{0}(z)$ - функция Бесселя нулевого порядка; $\mu_{n}-$ корни уравнения $J_{0}^{\prime}(\mu)=0$ или $J_{1}(\mu)=0$, а $A_{n}(t)$ и $B_{n}(t)$ - неизвестные функции, определяющие зависимость потенциалов от времени.

Пользуясь тем, что произвольную функцию можно разложить в ряд Дини-Бесселя, представим возмущение равновесной поверхности $\xi(r, t)$ в виде

$$
\xi(r, t)=C_{0}(t)+\sum_{n=1}^{\infty} C_{n}(t) \cdot J_{0}\left(\frac{\mu_{n}}{R} r\right) .
$$

Используя условие несжимаемости жидкости, можно показать, что $C_{0}(t)$ следует положить равным нулю. В результате выражение для возмущения свободной поверхности примет вид

$$
\xi(r, t)=+\sum_{m=1}^{\infty} C_{n}(t) J_{0}\left(\mu_{n} \frac{r}{R}\right) .
$$

Введем безразмерную переменную $x=\frac{r}{R}$, а в выражении (6) для $h(r)$ выделим коэффициент, зависящий от физических параметров, вводя безразмерную функцию $\chi(x)$ :

$$
h(r)=\frac{1}{2 \pi} \frac{q^{2}}{\rho g L^{4}} \chi(x), \chi(x)=\left(1+x^{2} \frac{R^{2}}{L^{2}}\right)^{-3} .
$$

Подставим выражения (10) и (12) в кинематическое условие (7) на свободной поверхности жидкости и, используя условие ортогональности функций Бесселя:

$$
\int_{0}^{1} J_{0}\left(\mu_{n} x\right) J_{0}\left(\mu_{k} x\right) x d x=\frac{1}{2}\left(J_{0}\left(\mu_{k}\right)\right)^{2} \delta_{n, k},
$$


получим связь коэффициентов разложений $B_{n}(t)$ и $C_{n}(t)$ :

$$
\begin{gathered}
C_{n}^{\prime}(t)=\frac{\mu_{n}}{R} B_{n}(t)+\frac{1}{4 \pi} \frac{q^{2}}{\rho g L^{4}} \sum_{m=1}^{\infty} \frac{\mu_{m}}{R} B_{n}(t) D_{m, n}, \\
D_{m, n}=\frac{4}{\left(J_{0}\left(\mu_{n}\right)\right)^{2}} \int_{0}^{1}\left(\mu_{m} \chi(x) J_{0}\left(\mu_{m} x\right)\right. \\
\left.+J_{1}\left(\mu_{m} x\right) \chi^{\prime}(x)\right) J_{0}\left(\mu_{n} x\right) x d x .
\end{gathered}
$$

Численные оценки показывают, что коэффициенты $D_{m, n}$ для $m=1$ по величине не превосходят единицы и быстро убывают с ростом $n$ (при $n=5$ их величина составляет сотые доли единицы).

Для оценки напряженности кулоновского поля, при которой реализуется неустойчивость поверхности жидкости, ввиду отсутствия более адекватных ситуации подходов введем для начала безразмерный полевой параметр:

$$
w_{0}=\frac{1}{4 \pi} \frac{q^{2}}{L^{4} \sqrt{\rho g \sigma}},
$$

характеризующий величину внешнего электрического поля у поверхности жидкости (впоследствии его скорректируем). Если в качестве характерного значения напряженности поля выбрать ее максимальное значение (непосредственно под точечным зарядом), т. е. $E \equiv q / L^{2}$, тогда введенный выше полевой параметр по аналогии с [4] может быть записан в виде

$$
w_{0}=\frac{1}{4 \pi} \frac{E^{2}}{\sqrt{g \rho \sigma}} .
$$

Используя понятие капиллярной постоянной жидкости $a=\sqrt{\sigma / \rho g}$, коэффициент, стоящий перед суммой в уравнении (14), приведем к виду

$$
\frac{1}{4 \pi} \frac{q^{2}}{\rho g L^{4} R}=w_{0} \frac{a}{R}
$$

Отметим, что отношение $\frac{a}{R}: 10^{-3}$. Кроме того, как следует из (13), этот коэффициент характеризует безразмерную величину функции, описывающей малое искажение равновесной поверхности. Поэтому уравнение (14) будем решать методом последовательных приближений.

В нулевом приближении пренебрегаем в (14) всей суммой, и найдем связь между коэффициентами $B_{n}$ и $C_{n}$ в виде

$$
B_{n}(t)=\frac{R}{\mu_{n}} C_{n}^{\prime}(t)
$$

В следующем приближении найденное в нулевом приближении выражение для коэффициента $B_{n}(t)$ используем под знаком суммы в (14) и найдем взаимосвязь между коэффициентами $B_{n}(t)$ и $C_{n}(t)$ с точностью до первого порядка по $\eta$

$$
B_{n}(t)=\frac{R}{\mu_{n}}\left(C_{k}^{\prime}(t)-\frac{w_{0} \cdot a}{R} \sum_{m=1}^{\infty} C_{m}^{\prime}(t) D_{m, n}\right) .
$$

Подставляя выражение (11) и (12) в условие эквипотенциальности (9), учитывая явный вид $\Phi^{(0)}(r, z)$ и вновь используя ортогональность функций Бесселя, получим выражение для коэффициентов $A_{n}(t)$ :

$$
\begin{gathered}
A_{n}(t)=-\frac{q}{L^{2}} \sum_{m=1}^{\infty} C_{m}(t) F_{m, m}, \\
F_{m, n}=\frac{4}{\left(J_{0}\left(\mu_{n}\right)\right)^{2}} \int_{0}^{1} \sqrt{\chi(x)} \cdot J_{0}\left(\mu_{m} x\right) J_{0}\left(\mu_{n} x\right) x d x .
\end{gathered}
$$

Из динамического условия (8), подставляя выражения (10)-(13), получим

$$
\begin{aligned}
& C_{n}^{\prime \prime}(t)+C_{n}(t) \frac{\mu_{n} g}{R}\left(1+\frac{\sigma}{g \rho} \frac{\mu_{n}^{2}}{R^{2}}\right) \\
& -w_{0} \frac{a}{R} \sum_{m=1}^{\infty}\left(C_{m}^{\prime \prime}(t)\left(D_{m, n}-G_{m, n}+w_{0} \frac{a}{R} \sum_{p=1}^{\infty} D_{m, p} G_{p, n}\right)\right. \\
& \left.+C_{m}(t) \frac{\mu_{n}}{R} g \sum_{p=1}^{\infty} \mu_{p} F_{m, p} F_{p, n}\right)=0 \\
& G_{m, n}=\frac{4}{\left(J_{0}\left(\mu_{n}\right)\right)^{2}} \int_{0}^{1} \mu_{n} \sqrt{\chi(x)} \cdot J_{0}\left(\mu_{m} x\right) \cdot J_{0}\left(\mu_{n} x\right) x d x .
\end{aligned}
$$

Это уравнение является эволюционным и описывает временную эволюцию амплитуд $C_{n}(t)$ в выражении для возмущения формы поверхности (12).

Решать эволюционное уравнение (17) будем методом последовательных приближений по величине коэффициента. Пренебрегая в $(17)$ слагаемыми $\sim(w \cdot a / R)^{2}$ и выделяя в сумме по $m$ слагаемое с $m=n$, получим

$$
\begin{aligned}
& C_{n}^{\prime \prime}(t)+C_{n}(t) \frac{\mu_{n}}{R} g\left(\left(1+\frac{\mu_{n}^{2}}{R^{2}} a^{2}\right)\right. \\
& +w_{0} \frac{a}{R}\left(\left(D_{n, n}-G_{n, n}\right)\left(1+\frac{\mu_{n}^{2}}{R^{2}} a^{2}\right)-\sum_{p=1}^{\infty} \mu_{p} F_{n, p} F_{p, n}\right) \\
& =w_{0} \frac{a}{R} \sum_{m=1}^{\infty}\left(1-\delta_{m, n}\right)\left(C_{m}^{\prime \prime}(t)\left(D_{m, n}-G_{m, n}\right)\right. \\
& \left.+C_{m}(t) \frac{\mu_{n}}{R} g \sum_{p=1}^{\infty} \mu_{p} F_{m, p} F_{p, n}\right) .
\end{aligned}
$$

Легко видеть, что (18) является системой связанных дифференциальных уравнений второго порядка. В нулевом приближении пренебрежем всеми слагаемыми, описывающими взаимодействие волн, т.е. отбросим правую 
часть уравнения. Уравнение (18) примет вид

$$
\begin{aligned}
& C_{n}^{\prime \prime}(t)+C_{n}(t) \frac{\mu_{n}}{R} g\left(\left(1+\frac{\mu_{n}^{2}}{R^{2}} a^{2}\right)+w_{0} \frac{a}{R}\right. \\
& \left.\times\left(\left(D_{n, n}-G_{n, n}\right)\left(1+\frac{\mu_{n}^{2}}{R^{2}} a^{2}\right)-\sum_{p=1}^{\infty} \mu_{p} F_{n, p} F_{p, n}\right)\right)=0 .
\end{aligned}
$$

Это гармоническое уравнение, решение которого ищем в виде $C_{n}(t)=\alpha_{n} \cdot \exp \left(i \omega_{n} t\right)$. Подставляя такой проект решения в (19), получим уравнение, связывающее частоты волн с $\mu_{n} / R$ - аналогом волнового числа:

$$
\begin{aligned}
& \omega_{n}^{2}=\frac{\mu_{n}}{R} g\left(\left(1+\frac{\mu_{n}^{2}}{R^{2}} a^{2}\right)+w_{0} \frac{a}{R}\right. \\
& \left.\times\left(\left(D_{n, n}-G_{n, n}\right)\left(1+\frac{\mu_{n}^{2}}{R^{2}} a^{2}\right)-\sum_{p=1}^{\infty} \mu_{p} F_{n, p} F_{p, n}\right)\right) .
\end{aligned}
$$

В первом приближении в (18) в слагаемых, учитывающих взаимодействие волн (т.е. в правой части уравнения), используем решение нулевого приближения $C_{m}(t)=\alpha_{m} \cdot \exp \left(i \omega_{m} t\right)$ и получим систему неоднородных уравнений

$$
\begin{aligned}
& C_{n}^{\prime \prime}(t)+C_{n}(t) \omega_{n}^{2}=w_{0} \frac{a}{R} \sum_{m=1}^{\infty}\left(1-\delta_{m, n}\right) \alpha_{m} \exp \left(i \omega_{m} t\right) \\
& \times\left(-\omega_{m}^{2}\left(D_{m, n}-G_{m, n}\right)+\frac{\mu_{n}}{R} g \sum_{p=1}^{\infty} \mu_{p} F_{m, p} F_{p, n}\right)=0 .
\end{aligned}
$$

Решение (21) ищется как суперпозиция общего решения однородных уравнений и частных решений неоднородных. Общее решение однородного уравнения имеет такой же вид, как и решение нулевого приближения

$$
C_{n}^{\text {hom }}(t)=\alpha_{n} \exp \left(i \omega_{n} t\right)
$$

Частное решение неоднородных уравнений (21) ищем в виде аналогичном виду функции неоднородности

$$
C_{n}^{n o n \text { hom }}(t)=\sum_{m=1}^{\infty}\left(1-\delta_{m, n}\right) \beta_{m} \exp \left(i \omega_{m} t\right) .
$$

Подставляем решение (23) в уравнение (21) и получаем

$$
\begin{aligned}
& \sum_{m=1}^{\infty}\left(1-\delta_{m, n}\right) \beta_{m} \exp \left(i \omega_{m} t\right)\left(\omega_{n}^{2}-\omega_{m}^{2}\right) \\
& =w_{0}^{2} \frac{a}{R} \sum_{m=1}^{\infty}\left(1-\delta_{m, n}\right) \alpha_{m} \exp \left(i \omega_{m} t\right) \\
& \times\left(-\omega_{m}^{2}\left(D_{m, n}-G_{m, n}\right)+\frac{\mu_{n}}{R} g \sum_{p=1}^{\infty} \mu_{p} F_{m, p} F_{p, k}\right)=0 .
\end{aligned}
$$

Используя ортогональность экспонент с мнимыми показателями, приравняем коэффициенты при одинаковых экспонентах в выражении (24) и найдем выражение для $\beta_{m}$ :

$$
\begin{aligned}
\beta_{m} & =w_{0} \frac{a}{R} \frac{\alpha_{m}}{\left(\omega_{m}^{2}-\omega_{n}^{2}\right)} \\
& \times\left(\omega_{m}^{2}\left(D_{m, n}-G_{m, n}\right)-\frac{\mu_{n}}{R} g \sum_{p=1}^{\infty} \mu_{p} F_{m, p} F_{p, n}\right) .
\end{aligned}
$$

Общее решение неоднородных уравнений (21) собирается из решений (22) и (23) с учетом коэффициентов в (25):

$$
\begin{aligned}
& C_{n}(t)=\alpha_{n} \exp \left(i \omega_{m} t\right)+w_{0} \frac{a}{R} \sum_{m=1}^{\infty}\left(1-\delta_{m, n}\right) \alpha_{m} \exp \left(i \omega_{m} t\right) \\
& \times \frac{1}{\omega_{m}^{2}-\omega_{n}^{2}}\left(\omega_{m}^{2}\left(D_{m, n}-G_{m, n}\right)-\frac{\mu_{n} g}{R} \sum_{p=1}^{\infty} \mu_{p} F_{m, p} F_{p, n}\right) .
\end{aligned}
$$

\section{Исследование полученного выражения для волнового возмущения равновесной поверхности}

Итак, форма свободной поверхности жидкости, возмущенной волновым движением осевой симметрии в поле точечного заряда, определяется следующим выражением:

$$
z=\frac{1}{2 \pi} \frac{q^{2}}{\rho g L^{4}}\left(1+\frac{r^{2}}{L^{2}}\right)^{-3}+\xi(r, t),
$$

где возмущение равновесной поверхности $\xi(r, t)$ представляется в виде разложения по корням уравнения $J_{1}(\mu)=0$ :

$$
\xi(r, t)=\sum_{n=1}^{\infty} C_{n}(t) J_{0}\left(\frac{\mu_{n}}{R} r\right) .
$$

Коэффициенты разложения $C_{n}(t)$ определяются выражением (26).

Легко заметить, что в отличие от тригонометрических функций расстояние между нулями функции Бесселя для фиксированного значения $\mu_{n} / R$ - величина переменная, и с увеличением отношения $\mu_{n} / R$ это расстояние уменьшается. Примем за аналог длины волны величину, равную расстоянию между двумя точками пересечения функции Бесселя $J_{0}\left(\frac{\mu_{n}}{R} r\right)$ с осью абсцисс, номера которых отличаются на две единицы. Получим следующее выражение:

$$
\lambda_{n, m}=\frac{R}{\mu_{n}}\left(v_{m+2}-v_{m}\right) ;(n, m=1,2,3 \ldots),
$$

где $v_{m}-$ корни уравнения $J_{0}(v)=0$. 


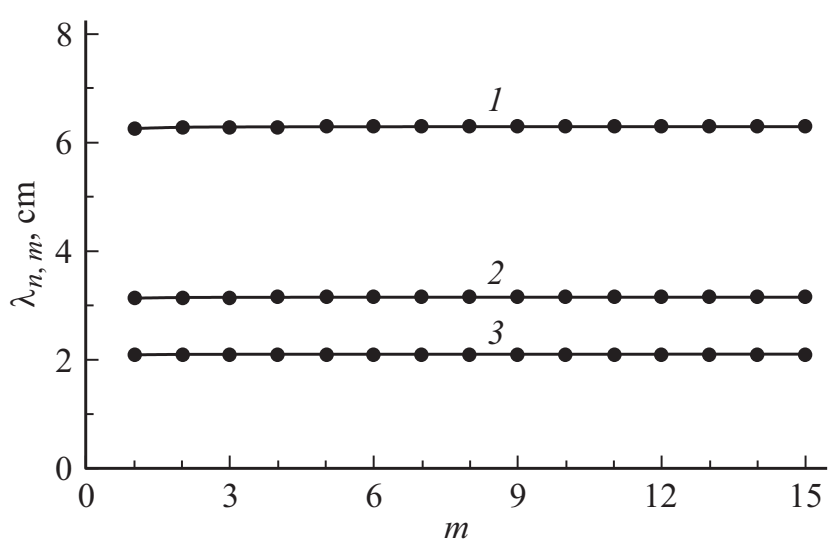

Рис. 2. Изменение радиуса кольцевых волн с ростом номеpa $m$ корня уравнения $J_{0}(v)=0$ по мере удаления от центра бассейна: $1-\frac{\mu_{n}}{R}=1 ; 2-\frac{\mu_{n}}{R}=2 ; 3-\frac{\mu_{n}}{R}=3$.

На рис. 2 представлены графики изменения величины $\lambda_{n, m}$ от номера $m$ для фиксированных значений отношения $R / \mu_{n}$, т. е. изменение $\lambda_{n, m}$ по мере удаления от начала координат. Видно, что сколь либо заметное изменение величины $\lambda_{n, m}$ происходит лишь вблизи центра возмущения. Таким образом, оперировать понятием длины волны допустимо и в случае кольцевых волн, но на некотором удалении от центра. Отношение $\mu_{n} / R$ в этом случае будет иметь смысл волнового числа.

Следует отметить, что на этом графике и всех нижеследующих расчеты для кольцевых волн проводились численно.

Сравним полученное выше решение с решением аналогичной задачи о кольцевых волнах на плоской безграничной поверхности бесконечно глубокой жидкости в однородном электрическом поле $E_{0}$, которое имеет вид [21]:

$$
\xi(r, t)=\int_{0}^{\infty} C_{k}(t) \cdot J_{0}(k r) d k, C_{k}(t)=\alpha_{k} \cdot \exp \left(i \omega_{k} t\right),
$$

где $k$ - аналог волнового числа, а частоты $\omega_{k}$ удовлетворяют уравнению (имеющему смысл дисперсионного):

$$
\omega^{2}=k-w_{E} k^{2}+k^{3}, w_{E}=\frac{1}{4 \pi} \frac{E_{0}^{2}}{\sqrt{g \rho \sigma}}
$$

Из сравнения выражений (26) и (27) для коэффициентов $C_{n}(t)$ и $C_{k}(t)$, описывающих временную эволюцию волнового возмущения поверхности, следует, что в отличие от случая однородного поля, в поле точечного заряда наблюдается взаимодействие волн различной длины. За это взаимодействие ответственно именно внешнее поле, поскольку слагаемые в выражении (26), описывающие взаимодействие, пропорциональны полевому параметру.

\section{Анализ дисперсионного уравнения}

Анализ уравнения (20) будем проводить численно, поскольку численные коэффициенты $G_{n, n}, D_{n, n}, F_{n, p}$ являются интегралами от функций Бесселя.

Соотношения (20),(21), (24)-(26) содержат бесконечную сумму. На рис. 3 приведены зависимости величин слагаемых этой суммы $\mu_{p} F_{n, p} F_{p, n}$ от порядкового номеpa $p$. Видно, что величина слагаемых быстро уменьшается с увеличением $p$. Поэтому для оценки значений суммы с заданной степенью точности достаточно учесть конечное число слагаемых (нижеследующие расчеты проводились с погрешностью менее $1 \%$ ).

Заметим, что, согласно (20), в отсутствии электрического поля, (когда $\left.w_{0}=0\right)$ все волны устойчивы: $\omega_{n}^{2}>0$, а сами частоты $\omega_{n}$ - вещественны для любого $n$. При увеличении значения полевого параметра $w$ квадрат частоты станет отрицательным $\omega_{n}^{2}<0$, следовательно, частота $\omega_{n}$ станет мнимой, и амплитуда соответствующей волны будет нарастать экспоненциально со временем, т. е. реализуется неустойчивость.

Критическое значение параметра $w_{0}^{*}$, разделяющее области устойчивых и неустойчивых волн, определяется условием $\omega_{n}^{2}=0$. Из (20) легко получить

$$
w_{0}^{*}=\frac{R}{a} \frac{\left(1+\mu_{n}^{2} a^{2} / R^{2}\right)}{\left(G_{n, n}-D_{n, n}\right)\left(1+\mu_{n}^{2} a^{2} / R^{2}\right)+\sum_{p=1}^{\infty} \mu_{p} F_{n, p} F_{p, n}} .
$$

На рис. 4, $а$ приведены нейтральные кривые (разделяющие устойчивые и неустойчивые состояния поверхности проводящей жидкости) для двух случаев: поля точечного заряда $w_{0}^{*}=w_{0}^{*}\left(\frac{\mu_{n}}{R}\right)$ (сплошная линия, рассчитанная численно по точкам из-за наличия бесконечной суммы с погрешностью порядка 1\%) и однородного электростатического поля $w_{E}^{*}=w_{E}^{*}(k)$ (штриховая линия, рассчитанная строго по аналитическому выражению [4]). Видно, что в области длинных волн

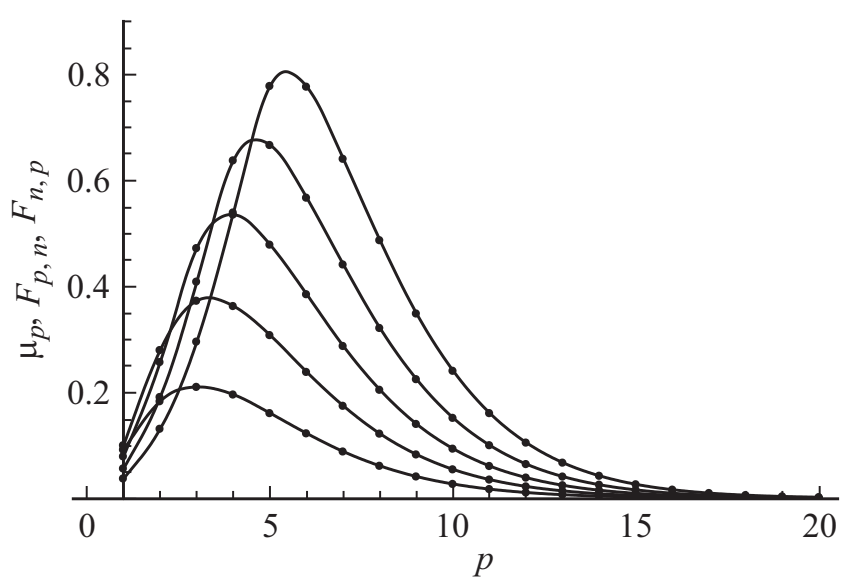

Рис. 3. Зависимость величин слагаемых $\mu_{p} F_{n, p} F_{p, n}$ от порядкового номера $p$. Линии снизу вверх соответствуют значениям $n=1,2,3,4,5$. 



Рис. 4. Сравнение нейтральных кривых: сплошная линия соответствует полю точечного заряда, штриховая - однородному полю. $a$ - полевой параметр определен соотношением (15); $b$ - полевой параметр определен соотношением (28).

(малые значения волновых чисел) нейтральная кривая для случая однородного поля лежит ниже аналогичной кривой для случая поля точечного заряда. В области коротких волн (большие значения волновых чисел) ситуация противоположна.

Зоны устойчивости для поля точечного заряда при $k$, $\frac{\mu_{n}}{R}<4.5$ лежат выше соответствующих зон для однородного электростатического поля. Причина этого в некорректном определении параметра для случая поля точечного заряда: в случае однородного электростатического поля условия, критические для реализации неустойчивости, выполняются сразу для всей поверхности жидкости, а для поля точечного заряда они выполнятся лишь для одной точки, лежащей точно под зарядом. Если принять за основу критические условия реализации неустойчивости Тонкса-Френкеля (строго теоретическим путем найденные в [4]), то введенный соотношением (15) полевой параметр $w_{0}$ следует уменьшить в 4 раза. В этом случае результаты проведенного выше расчета критических условий реализации неустойчивости кольцевых волн в неоднородном поле совпадут (в минимуме) с критическими условиями неустойчивости одномерных плоских волн, а также кольцевых волн в однородном электростатическом поле, и примут вид

$$
w=\frac{1}{16 \pi} \frac{E^{2}}{\sqrt{g \rho \sigma}},
$$

где $E \equiv\left(q / L^{2}\right)-$ напряженность кулоновского поля на поверхности жидкости прямо под зарядом.

Следует отметить, что в кулоновском поле при наступлении критических условий будут терять устойчивость кольцевые волны на поверхности жидкости в малой окрестности центра (на оси симметрии задачи). Согласно фотографиям, приведенным в [2], с увеличением напряженности поля у поверхности жидкости площадь поверхности жидкости, охваченной неустойчивостью, растет. В то время как неустойчивость ТонксаФренкеля (одномерные волны) реализуется сразу на всей поверхности жидкости.

Зависимости, аналогичные приведенным на рис 4, $a$, но с переопределенным (28) значением полевого параметра для точечного заряда, приведем на рис. $4, b$. Видно, что искомые зависимости, практически совпадают (с учетом погрешности приближенных расчетов для кольцевых волн) в окрестности минимума кривых (при $\left.\left(\mu_{n} / R\right) \approx k \approx 1\right)$, но расходятся при $\left(\mu_{n} / R\right)>1.2$ и $k>1.2$. Видно, что в этой области неустойчивость кольцевых волн начинается при меньших значениях напряженности поля у поверхности жидкости.

Из рисунков видно, что для начала реализации неустойчивости поверхности жидкости в неоднородном поле (точечный заряд, диполь, стержень, поддерживаемый при постоянном потенциале) нужны меньшие значения напряженности поля на поверхности жидкости непосредственно под зарядом (диполем или стержнем).

Их общефизических соображений можно сказать, что в неоднородном осесимметричном электростатическом поле неустойчивость сначала реализуется в центре (под зарядом, диполем или стержнем), и лишь при увеличении напряженности распространяется на большую площадь.

Как уже говорилось выше, в области неустойчивости квадрат частоты становится отрицательным, а инкремент неустойчивости определится выражением $\gamma_{n} \equiv \sqrt{-\omega_{n}^{2}}$.

Изменение инкремента с ростом $\mu_{n} / R$ носит немонотонный характер и имеет максимум. Увеличение полевого параметра приводит к увеличению инкрементов и смещению волнового числа наиболее неустойчивой волны в область больших значений.

На рис. 5 представлены инкременты в зависимости от $\mu_{n} / R$ и $k$ для случаев поля точечного заряда и однородного поля. Легко заметить, что инкременты в случае однородного поля существенно выше инкрементов для поля точечного заряда. Области неустойчивых волновых чисел также существенно шире в случае однородного поля. 


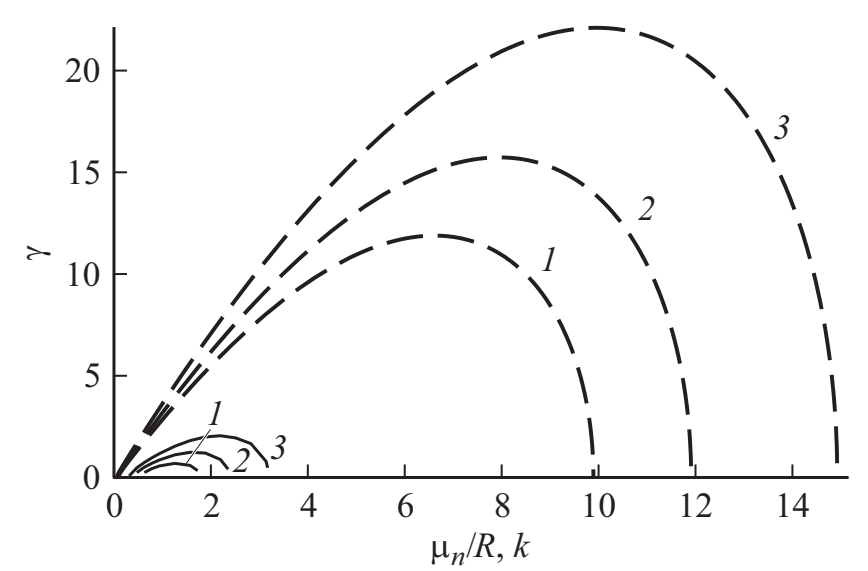

Рис. 5. Значения инкрементов в зависимости от волнового числа или его аналога: сплошные линии - кольцевые волны в поле точечного заряда, штриховые линии - одномерные волны в однородном поле: $1-w=10 ; 2-w=12 ; 3-w=15$.

Полученный результат: то, что в случае неоднородного поля критические условия начала реализации неустойчивости неоднородно индукционно заряженной поверхности жидкости отличаются от таковых для случая однородного электростатического поля можно прокомментировать и с энергетической точки зрения. Для однородного поля критические условия выполняются одновременно для всей поверхности, для чего плотность энергии поля у поверхности жидкости должна достичь критического значения на всей поверхности. Для неоднородного же поля критические условия должны сначала выполниться только для малой окрестности начала координат, и плотность энергии неоднородного поля должна достичь критического значения только в его окрестности. По мере увеличения напряженности неоднородного поля неустойчивость будет охватывать все большую площадь в окрестности центра, что и наблюдается в эксперименте [2]. Сама плотность энергии электростатического поля должна быть достаточна для совершения работы против поля сил тяжести и увеличения площади поверхности жидкости, при росте конусов Тейлора на стадии реализации неустойчивости поверхности жидкости [22] по отношению к индукционному заряду.

Следует также отметить, что хотя мы говорим об электростатической неустойчивости кольцевых волн, она реализуется в виде одиночных выступов (конусов Тейлора [22]), расположенных по кругу, как это и наблюдается в экспериментах $[1,2]$. Собственно говоря, такая же картина: разбиения сплошной волны на отдельные выступы наблюдается и для одномерных плоских волн. При этом явлении потенциальная энергия сил поверхностного натяжения уменьшается, т.е. процесс разбиения сплошной волны на совокупность отдельных выступов энергетически выгоден, также как при распаде жидкого цилиндра на отдельные капли [23].

\section{Какие эксперименты желательно провести}

На данном этапе исследования ощущается нехватка экспериментальных данных. Необходимо провести экспериментальные исследования закономерностей реализации неустойчивости кольцевых волн на поверхности проводящей жидкости (в $[1,2]$ эксперименты проводились с плохо проводящими жидкостями) в цилиндрическом бассейне в однородном и неоднородных (точечный заряд, диполь, стержень, поддерживаемый при постоянном потенциале) электростатических полях с целью определения критических условий реализации неустойчивости кольцевых волн.

Следует отметить, что исследование неустойчивости однородно заряженной поверхности жидкости по отношению к одномерным плоским волнам началось с теории $[3,4]$, и только впоследствии были проведены качественные эксперименты [5]. Эксперименты Тейлора [22], хотя и проведены аккуратно, но содержат мало фактического материала о реализации неустойчивости. Рассматриваемая ситуация с двумерными кольцевыми волнами более сложна в теоретическом отношении, и теоретику желательно иметь в своем распоряжении несколько экспериментальных фактов. Так, неплохо бы знать, каким образом реализуется неустойчивость по мере увеличения напряженности неоднородного поля у поверхности жидкости. Общие физические соображения говорят о том, что сначала неустойчивость должна реализоваться в центре бассейна, там, где напряженность поля максимальна. Но неустойчивость не может реализоваться в точке, должна быть задействована какаято площадь поверхности жидкости в ее окрестности. Каким будет радиус круга, в котором реализуется неустойчивость в виде одиночного конуса Тейлора? В какой очередности будет реализовываться неустойчивость кольцевых волн при увеличении напряженности?

\section{Заключение}

Исследованы кольцевые волны в неоднородном кулоновском поле. Построена нейтральная кривая, разделяющая области устойчивых и неустойчивых волн. Показано, что в поле точечного заряда наблюдается взаимодействие волн различной длины. Найдено, что критические условия реализации неустойчивости коротких кольцевых волн различной длины в случаях поля точечного заряда и однородного электростатического поля различны. Величины инкрементов неустойчивых волн в поле точечного заряда существенно ниже по сравнению с однородным электростатическим полем.

\section{Конфликт интересов}

Авторы заявляют, что у них нет конфликта интересов. 


\section{Список литературы}

[1] Bychkov V.L., Ershov A.P., Chernikov V.A. Corona discharge modeling of some ball lightning features // Atmosphere, ionosphere, safety. Proceedings of 10-th International symposium on Ball Lightning. 2008. July 7-12. P. 7-11.

[2] Саранин В.А., Майер В.В., Вараксина Е.И. // ЖЭТФ. 2014. T. 146. № 2(8). C. 398-404.

[3] Tonks L. // Phys. Rev. 1935. Vol. 48. P. 562-568.

[4] Френкель Я.И. // ЖЭТФ. 1936. Т. 6. № 4. С. 348-350.

[5] Габович М.Д. // УФН. 1983. Т. 140. № 1. С. 137-151.

[6] Le Mehaute B. // J. Fluid. Mech. 1988 Vol. 197. P. 415-427.

[7] Craye C., Sobieski P.V., Bliven L.F., Guissard A. // IEEE J. Oceanic Eng. 1999. Vol. 24. N 3. P. 323-332.

[8] Kartashov Ya.V., Visloukh V.A., Torner L. // Opt. Express. 2007. Vol. 15. N 24. P. 16216-16221.

[9] Чашечкин Ю.Д., Прохоров В.Е. // ДАН. 2013. Т. 451. № 1. C. 41-45.

[10] Bahni Ray, Gautam Biswas, Ashutosh Sharm // J. Fluid. Mech. 2015. Vol. 768. P. 492-523.

[11] Nils Paul van Hinsberg, Marie CharbonneauGrandmaison // Phys. Rev. E. 2015. Vol. 92. P. 013004 $1-12.9$.

[12] Григорьев А.И. // ЖТФ. 2007. Т. 77. Вып. 2. С. 31-40.

[13] Кочин Н.Е., Кибель И.А., Розе Н.В. Теоретическая механика. Ч. 1. М.: Физматиздат, 1963. 584 с.

[14] Кошляков Н.С., Глинер Э.Б., Смирнов М.М. Уравнения в частных производных математической физики. М.: Высшая школа, 1970. $712 \mathrm{c}$.

[15] Безручко Б.П., Диканев Т.В., Захаревич А.М. Гравитационно-капиллярные волны на поверхности жидкости. Саратов: Изд-во ГосУНЦ Колледж, 2003. 17 с.

[16] Сретенский Л.Н. Теория волновых движений жидкости. М.: Наука, 1977. 815 с.

[17] Черкесов Л.В. Введение в гидродинамику и теорию волн. СПб.: Гидрометеоиздат, 1992. 264 с.

[18] Батыгин В.В., Топтыгин И.Н. Сборник задач по электродинамике. М.: Наука, 1970. 503 с.

[19] Ландау Л.Д., Лифиии Е.М. Теоретическая физика. Т. 6. Гидродинамика. М.: Наука, 1986. 736 с.

[20] Ландау Л.Д., Лифиии Е.М. Теоретическая физика. Т. 2. Теория поля. М.: Наука, 1973. 504 с.

[21] Shiryaeva S.O., Grigor'ev A.I., Orlova K.I. // Surf. Eng. Appl. Electrochem. 2018. Vol. 54. N. 4. P. 374-378.

[22] Tailor G.I., McEwan A.D. // J. Fluid. Mech. 1965. Vol. 22. N 1. P.1-15.

[23] Ширяева С.О., Григорьев А.И. Спонтанный распад струй. Ярославль: Изд-во ЯрГУ им. П.Г. Демидова, 2012. 204 с. 